\title{
Dinâmica e contribuição da micorriza arbuscular em sistemas de produção com rotação de culturas
}

\author{
Jeanne Christine Claessen de Miranda ${ }^{(1)}$, Lourival Vilela ${ }^{(1)}$ e Leo Nobre de Miranda(1)
}

(1)Embrapa Cerrados, BR 020, Km 18, Rodovia Brasília-Fortaleza, Caixa Postal 08223, CEP 73310-970 Planaltina, DF. E-mail: jeanne@cpac.embrapa.br, Ivilela@cpac.embrapa.br, leo@cpac.embrapa.br

\begin{abstract}
Resumo - Rotação de culturas e variações sazonais podem promover alterações quantitativas e qualitativas na comunidade de fungos micorrízicos arbusculares nativos e na formação da micorriza arbuscular. Essa dinâmica foi avaliada, em campo, num Latossolo Vermelho, em relação ao tempo de cultivo e variação sazonal, em sistemas de rotação de culturas. Em casa de vegetação, avaliou-se, em solo proveniente da área experimental, a contribuição da micorriza arbuscular no crescimento de soja e capim-andropógon utilizados na rotação. O número de esporos dos fungos aumentou no solo cultivado. O número de esporos e o porcentual de colonização radicular, inicialmente maiores sob pastagem, variaram de acordo com o tempo de cultivo, as estações seca e chuvosa, a cultura e o sistema de rotação utilizados. O número de gêneros e espécies aumentou com o tempo de cultivo e manejo de culturas e foi maior sob culturas anuais em rotação. A presença dos fungos no solo contribuiu no crescimento da soja e do capim-andropógon em 53\% e 95\%, respectivamente. A cultura e o sistema de cultivo são fatores determinantes para o enriquecimento do sistema com micorriza arbuscular.
\end{abstract}

Termos para indexação: fungos micorrízicos arbusculares, esporos, colonização radicular, culturas anuais, pastagens.

\section{Dynamics and contribution of arbuscular mycorrhiza in culture systems with crop rotation}

\begin{abstract}
Crop rotation and seasonal variations can promote quantitative and qualitative changes in the indigenous arbuscular mycorrhizal fungi population in the soil and arbuscular mycorrhiza establishment. These fungi dynamics were evaluated in the field, in a Red Latosol, in relation to cropping time, seasonal variation and rotation systems. The contribution of arbuscular mycorrhiza to the growth of andropogon grass and soybean, which were used in the systems, was evaluated in a greenhouse experiment using soil from the experimental area. The number of spores of the fungi increased in the cultivated soil. The spores number and percent root colonization varied according to cropping time, soil moisture, crops and rotation system and were, initially, higher under pasture. The number of genus and species increased according to cropping time and culture management and was higher under annual crops in rotation. The presence of these fungi in the soil contributed $53 \%$ and $95 \%$ to the growth of soybean and andropogon grass, respectively. Crops and cultivation system are crucial for the system's enrichment in arbuscular mycorrhiza.
\end{abstract}

Index terms: arbuscular mycorrhizal fungi, spores, root colonization, annual crops, pastures.

\section{Introdução}

O cultivo de plantas envolve a utilização de práticas agrícolas adequadas para a obtenção de retornos econômicos dos investimentos em insumos utilizados nos sistemas de produção. Os fungos micorrízicos arbusculares são componentes naturais desses sistemas e, por meio da micorriza arbuscular, podem aumentar a absorção de nutrientes do solo, como o fósforo. Essa contribuição é particularmente importante nos solos ácidos e de baixa fertilidade, como os do cerrado (Siqueira \& Moreira, 1997).

Os efeitos benéficos da calagem, adubação, rotação de culturas e dos adubos verdes, na propagação dos fungos micorrízicos no solo e na planta têm sido demonstrados em diversos trabalhos, e a execução adequa- 
da dessas práticas é especialmente importante, quando a comunidade nativa é deficiente em quantidade e em diversidade de espécies (Sieverding, 1991). Os fungos micorrízicos arbusculares diferem na maneira e na intensidade com que colonizam as raízes, e a alteração das condições do solo pode modificar a composição das espécies de fungo presentes durante a formação da micorriza em campo (Sieverding, 1991; Abbott \& Gazey, 1994). Essa composição da comunidade fúngica micorrízica tem, por sua vez, o potencial de influenciar (Heijden et al., 1998) e ser influenciada (Bever et al., 1996) pela composição da comunidade vegetal. Essa interação é relevante para os agroecossistemas, sobretudo aqueles que envolvem rotação de culturas e culturas intercalares.

Abbott \& Robson (1994) mostram que a rotação de culturas e as práticas de fertilização são determinantes para assegurar a abundância micorrízica. Segundo Bever et al. (1996), a planta hospedeira pode ser um dos principais fatores que regulam a composição e a estrutura das comunidades de fungos micorrízicos arbusculares, pois cada fase de seu desenvolvimento, como germinação de esporos, crescimento das hifas, colonização radicular e esporulação, é influenciada pelas raízes das plantas. De modo geral, as culturas apresentam diferentes graus de dependência micorrízica (Howeler et al., 1987; Hartnett \& Wilson, 2002) e especificidade, em relação à espécie do fungo micorrízico arbuscular e, conseqüentemente, alteram a densidade de inóculo e a ocorrência de espécies de fungos micorrízicos arbusculares presentes no solo (Bever et al., 1996; Eom et al., 2000). Como as espécies desses fungos não são igualmente eficientes para o crescimento das plantas (Heijden et al., 1998; Miranda \& Miranda, 2001), as alterações na composição da comunidade, assim como na abundância da micorriza, podem alterar a contribuição dos fungos micorrízicos arbusculares em geral (Heijden et al., 1998; Bever, 2002).

O objetivo deste trabalho foi avaliar a dinâmica, quantitativa e qualitativa, dos fungos micorrízicos arbusculares, em campo, em relação ao tempo de cultivo, em sistemas de produção com diversas culturas anuais e pastagens em rotação. Foi avaliada, também, em casa de vegetação, em solo proveniente dos tratamentos em campo, a contribuição desses fungos nativos no crescimento da soja (Glycine max (L.) Merrill) e do capim-andropógon (Andropogon gayanus cv. Planaltina), que foram utilizados na rotação, nas condições de campo.

\section{Material e Métodos}

As avaliações, em campo, foram conduzidas por seis anos consecutivos, na área experimental da Embrapa Cerrados, Planaltina, DF, em um ensaio instalado desde novembro de 1991, em Latossolo Vermelho argiloso, com o objetivo de avaliar a integração lavoura/pastagem.

Os tratamentos, estabelecidos em parcelas de 200x40 m, eram constituídos de cinco sistemas de plantio: lavoura anual contínua; pastagem de gramínea pura contínua; pastagem consorciada contínua; rotação de cultura anual/pastagem consorciada; rotação de pastagem consorciada/cultura anual. Áreas adjacentes com vegetação nativa de cerrado foram utilizadas para avaliação da comunidade de fungos micorrízicos arbusculares, nas condições originais do solo. No sistema contínuo de lavoura, a seqüência de culturas anuais foi: soja (Glycine $\max ($ L.) Merrill) cv. Doko (1992), soja cv. Siriema (1993), milho (Zea mays L.) híbrido BR 201 (1994), soja cv. Savana (1995) e milho híbrido BR 201 (1996). A densidade de semeadura foi de 380.000 plantas ha-1, de soja, com espaçamento entre linhas de $0,45 \mathrm{~m}$, e de 55.000 plantas ha $^{-1}$, de milho, com espaçamento entre linhas de 0,90 m. No sistema com pastagem pura, foi cultivado o Andropogon gayanus Kunth, var. bisquanulatus cv. Planaltina na densidade de $48 \mathrm{~kg} \mathrm{ha}^{-1}$ de sementes, com valor cultural de $8,7 \%$ e, na pastagem consorciada, foi utilizada essa forrageira com um coquetel de leguminosas-Calopogonium mucunoides, Stylosanthes guianensis cv. Mineirão, Centrosema brasilianum CPAC1219 e Neonotonia wightii cv. Comum, na proporção de 4,6:1,0:3,0:3,2 (2,3kg/0,5kg/1,5 kg/1,6 kg, respectivamente) -, e a densidade de semeadura foi de $5,9 \mathrm{~kg} \mathrm{ha}^{-1}$ de sementes. $\mathrm{O}$ objetivo do coquetel era garantir uma população de plantas que permitisse manter uma proporção de leguminosas de $30 \%$ a $40 \%$. Nos tratamentos com rotação, foi proposto um ciclo inicial de quatro anos, antes da primeira rotação a fim de permitir a geração de dados em número suficiente, para descrever as variações de interesse.

A correção da fertilidade do solo foi estabelecida de acordo com a análise do solo (Embrapa, 1979), para permitir o crescimento ótimo das plantas. Nas áreas com pastagens, a correção foi a seguinte: calagem com 5,8 t ha ${ }^{-1}$ de calcário PRNT 100\%, para atingir 50\% de saturação por bases; adubação de estabelecimento com $90 \mathrm{~kg} \mathrm{ha}^{-1}$ de $\mathrm{P}_{2} \mathrm{O}_{5}$, na forma de superfosfato simples, $120 \mathrm{~kg} \mathrm{ha}^{-1}$ de $\mathrm{K}_{2} \mathrm{O}$, na forma de cloreto de potássio, e $30 \mathrm{~kg} \mathrm{ha}^{-1}$ de FTE BR 12; adubação bienal de manutenção 
de $\mathrm{P}$ e $\mathrm{K}$ com $20 \mathrm{~kg} \mathrm{ha}^{-1}$ de $\mathrm{P}_{2} \mathrm{O}_{5}$, na forma de superfosfato simples, e $36 \mathrm{~kg} \mathrm{ha}^{-1}$ de $\mathrm{K}_{2} \mathrm{O}$, na forma de cloreto de potássio. Nas áreas com culturas anuais foi efetuada a seguinte correção: calagem com $5,8 t^{\text {ha }}{ }^{-1}$ de calcário PRNT 100\%, para atingir 50\% de saturação por bases e 2,8 t ha-1 de gesso agrícola; adubação de estabelecimento, com $490 \mathrm{~kg} \mathrm{ha}^{-1}$ da fórmula 0-20-20, $63 \mathrm{~kg} \mathrm{ha}^{-1}$ de FTE BR 12, soja com inoculação de Bradyrhizobium japonicum, e $80 \mathrm{~kg} \mathrm{ha}^{-1}$ de $\mathrm{N}$ na forma de uréia para o milho; adubação anual de manutenção com $490 \mathrm{~kg} \mathrm{ha}^{-1}$ da fórmula 0-20-20 e $80 \mathrm{~kg} \mathrm{ha}^{-1}$ de $\mathrm{N}$, na forma de uréia, para o milho. Nos tratamentos com lavoura, foi utilizado o preparo do solo convencional (aração e gradagem), anualmente, e pousio após a colheita, sem plantas de cobertura no período seco.

$\mathrm{O}$ ensaio instalado em campo foi um delineamento experimental de blocos ao acaso, com duas repetições. Entretanto, nas análises quantitativa e qualitativa da comunidade de fungos micorrízicos arbusculares foram coletadas amostras de solo e raízes, compostas de oitenta subamostras ( 1 subamostra/100 m²), em cada parcela, na profundidade de $0-20 \mathrm{~cm}$. Essas amostragens foram executadas, bianualmente, nas épocas seca e chuvosa, no período de floração das culturas anuais, e nos mesmos pontos de amostragem, a cada ano. Foram avaliados o número de esporos no solo (Gerdemann \& Nicolson, 1963; Coolen, 1979) e a colonização radicular das plantas (Phillips \& Hayman, 1970; Giovannetti \& Mosse, 1980), em todos os tratamentos. Nas áreas com rotação lavoura/pastagem e vice-versa, esses parâmetros foram avaliados somente no período chuvoso. A diversidade de espécies de fungos micorrízicos arbusculares presentes no solo foi avaliada pela análise morfológica de esporos isolados, das amostras de campo, e por comparação com esporos frescos provenientes de culturas armadilhas, estabelecidas conforme Morton et al. (1993), originadas dos mesmos pontos de amostragem em campo e conduzidas por quatro meses, utilizando-se o sorgo (Sorghum bicolor cultivar BR 300) e o Stylosanthes guianensis cultivar Mineirão, em consórcio, como plantas armadilhas. Foram utilizadas duas plantas, de maneira a eliminar os efeitos seletivos de espécies de plantas armadilhas, na estrutura da comunidade dos fungos micorrízicos arbusculares. As descrições originais de Schenck \& Pérez (1990) foram utilizadas para a identificação das espécies.

No período chuvoso de 1996 conduziu-se, em casa de vegetação, um experimento para avaliar o potencial de contribuição da micorriza arbuscular, nas condições praticadas em campo, no crescimento de plantas que foram utilizadas nos sistemas de plantio. Utilizou-se solo coletado nas parcelas das respectivas áreas experimentais, de sistemas contínuos de pastagem pura e lavoura, na profundidade de $0-20 \mathrm{~cm}$. Foram coletadas amostras, compostas de dez subamostras (constituídas, por sua vez, de 8 subsubamostras, num total de 80 amostragens por parcela), de $10 \mathrm{~kg}$ de solo por parcela. Em cada repetição de cada tratamento, metade da amostra foi mantida em condições naturais, enquanto a outra metade foi parcialmente esterilizada a vapor, por duas vezes, a $90^{\circ} \mathrm{C}$, por duas horas, com um intervalo de 24 horas entre os ciclos. Porém, nesses tratamentos, além da presença de esporos mortos dos fungos micorrízicos arbusculares nativos, fez-se, também, a reposição da microbiota do solo, mediante aplicação de uma suspensão não-micorrízica do solo natural (Koide \& Li, 1989) de cada parcela. O solo proveniente da área com pastagem pura foi cultivado com Andropogon gayanus cultivar Planaltina, por 60 dias, e o da área com lavoura foi cultivado com soja cultivar Savana, por 45 dias. Foram determinados a produção de matéria seca, o número de esporos no solo, a colonização radicular e a dependência micorrízica das plantas. O delineamento experimental foi o inteiramente ao acaso, com duas repetições por tratamento, mantendo-se o delineamento experimental praticado em campo. Os dados foram analisados estatisticamente pelos procedimentos do SAS (SAS Institute, 1989), e os dados de número de esporos no solo foram transformados em $(x+0,5)^{0,5}$ e de colonização radicular em arc sen $(x / 100)^{0,5}$.

\section{Resultados e Discussão}

O total de esporos de fungos micorrízicos arbusculares, produzido nos diferentes tratamentos, e a colonização radicular das plantas nos diferentes sistemas de cultivo e rotação são apresentados na Tabela 1 . Ao fazer uma avaliação da diferenciação da comunidade de fungos micorrízicos arbusculares em duas gerações de cultivo, por meio da contagem de esporos, Bever (2002) demonstrou que a contagem de esporos na primeira geração de cultivo reflete, efetivamente, os níveis de crescimento da população.

A densidade de esporos dos fungos micorrízicos arbusculares no solo de cerrado nativo foi, em geral, baixa e aumentou em função do cultivo das plantas, de forma semelhante ao observado nos solos, igualmente 
ácidos e inférteis, das savanas colombianas (Howeler et al., 1987; Dodd et al., 1990b). Verificou-se, também, que nos sistemas de produção com cultivo contínuo de pastagens e culturas anuais, essa densidade variou significativamente em função da cultura utilizada, da umidade do solo no período chuvoso e seco e do tempo de cultivo. Segundo Abbott \& Robson (1994) e Bever et al. (1996), a cultura utilizada é o fator de maior influência na ocorrência e abundância relativa dos fungos micorrízicos arbusculares no solo. Eom et al. (2000) sugerem que essas variações podem ser geradas pelas alterações, mediadas pela planta hospedeira, do microhabitat do solo e pelas variações na fenologia e dependência micorrízica das espécies hospedeiras, entre outros.

No período chuvoso, no primeiro ano de cultivo, em 1992, os maiores índices de esporos no solo ocorreram nas áreas com pastagens puras e consorciadas. Nessas áreas, houve aumento no número de esporos no solo, respectivamente, de 19 e 23 vezes, em relação ao cerrado nativo, ao passo que, nas áreas com culturas anuais, o incremento foi de apenas três vezes (Tabela 1). Oehl et al. (2003) observaram que a abundância de esporos no solo foi mais elevada em solo sob pastagens contínuas e mais baixo nas áreas com manejo mais intensivo do solo e cultivadas com monocultura de milho.

No primeiro período seco, em 1992, o número de esporos decresceu, significativamente, nas áreas com pastagens, e confirmou, assim, a indicação de outros autores de que, nas savanas tropicais, os períodos secos podem reduzir significativamente o número de esporos nas camadas superiores do solo (Howeler et al., 1987; Dodd et al., 1990b). Entretanto, esse decréscimo foi menor nas áreas com pastagem consorciada do que nas áreas com gramínea pura, o que mostra o efeito significativo da presença de leguminosas na manutenção da comunidade dos fungos micorrízicos arbusculares no solo. Esse efeito benéfico das leguminosas no potencial de inóculo do solo, em pastagens consorciadas com Andropogon gayanus, foi igualmente observado por Howeler et al. (1987) ao compararem diferentes gramíneas forrageiras e leguminosas isoladamente ou em consórcio. Eom et al. (2000) sugerem que esse aumento na abundância dos fungos micorrízicos arbusculares no solo, pelas leguminosas, pode ser atribuido à alta concentração de nitrogênio no solo rizosférico das plantas ou a benefícios recíprocos entre o rizóbio e os fungos. Além disso, a predominância do Stylosanthes guianensis cultivar Mineirão no consórcio e a sua alta retenção de folhas verdes durante a seca (Embrapa, 1998), assim como a continuidade das atividades fisiológicas dessas plantas, nesse período, podem ter contribuído para manter a associação micorrízica ativa. Na área cultivada com soja não houve, inicialmente, diferenças significativas entre o período chuvoso e seco.

Tabela 1. Esporos de fungos micorrízicos arbusculares nativos, nos períodos chuvoso e seco, e colonização radicular das plantas hospedeiras, no período chuvoso, em solo com uso contínuo de pastagem pura (PP), pastagem consorciada (PC), lavoura (LA) de soja (S) e milho (M) em rotação e no cerrado nativo (CN) ${ }^{(1)}$.

\begin{tabular}{|c|c|c|c|c|c|c|c|}
\hline \multirow[t]{2}{*}{ Ano } & \multicolumn{4}{|c|}{ Esporos no solo $\left(\mathrm{n}^{\circ} / 50 \mathrm{~g}\right)$} & \multicolumn{3}{|c|}{ Colonização radicular (\%) } \\
\hline & PP & $\mathrm{PC}$ & LA & $\mathrm{CN}$ & PP & $\mathrm{PC}$ & LA \\
\hline 1991 (Inicial) $^{(2)}$ & 15 & 12 & 10 & 12 & $\ldots^{(3)}$ & $\ldots$ & $\ldots$ \\
\hline & \multicolumn{7}{|c|}{ Período chuvoso (janeiro) } \\
\hline 1992 & 289 & 278 & $32 \mathrm{~S}$ & $\ldots$ & 69 & 74 & $30 \mathrm{~S}$ \\
\hline 1993 & 67 & 132 & $97 \mathrm{~S}$ & $\ldots$ & 28 & 32 & $38 \mathrm{~S}$ \\
\hline 1994 & 58 & 74 & $62 \mathrm{M}$ & 38 & 52 & 61 & $84 \mathrm{M}$ \\
\hline 1995 & 41 & 39 & $50 \mathrm{~S}$ & 10 & 28 & 34 & $62 \mathrm{~S}$ \\
\hline \multirow[t]{2}{*}{1996} & 29 & 35 & $61 \mathrm{M}$ & 2 & 51 & 50 & $84 \mathrm{M}$ \\
\hline & \multicolumn{7}{|c|}{ Período seco (setembro) } \\
\hline 1992 & 48 & 115 & 33 & $\ldots$ & $\ldots$ & $\ldots$ & $-(4)$ \\
\hline 1993 & 49 & 52 & 63 & 26 & 28 & 19 & - \\
\hline 1994 & 38 & 51 & 57 & 8 & 43 & 56 & - \\
\hline 1995 & 29 & 36 & 54 & 4 & 40 & 36 & - \\
\hline 1996 & 23 & 17 & 18 & $\ldots$ & $\ldots$ & $\ldots$ & - \\
\hline
\end{tabular}

(1)Os dados de esporos no solo foram transformados em $(x+0,5)^{0,5}$ e de colonização radicular em arc sen (x/100) ${ }^{0,5}$; a diferença mínima significativa a 5\% e o coeficiente de variação (\%) para colonização radicular, no perído chuvoso foi 10 e 9,6, respectivamente; a diferença mínima significativa a 5\% e o coeficiente de variação (\%) para número de esporos no solo, nos períodos chuvoso e seco, e chuvoso foi 33 e 13,2 respectivamente. ${ }^{(2) D e t e c t a d o ~ n a s ~ a ́ r e a s ~ e m ~ s e t e m b r o, ~ a p o ́ s ~ a ~ r e m o c ̧ a ̃ o ~ d a ~ v e g e t a c ̧ a ̃ o ~ n a t i v a . ~}{ }^{(3)}$ Não avaliado. ${ }^{(4)}$ Em pousio. 
Nos anos de 1994 a 1996, o número de esporos nas pastagens, no período chuvoso, diminuiu gradativamente a níveis similares aos observados na área com lavoura. Essa redução gradativa, após o primeiro ano, pode ocorrer em virtude do menor estresse decorrente da menor intensidade de preparo do solo, nos anos subseqüentes ao estabelecimento das pastagens, assim como da dependência temporária dessas plantas em relação à micorriza arbuscular, para o seu crescimento inicial. Dodd et al. (1990b) observaram, também, uma redução e uma tendência de estabilização no número de esporos no solo, com o tempo de cultivo de braquiária (Brachiaria dictyoneura) pura ou consorciada com kudzu tropical (Pueraria phaseoloides).

Na lavoura anual ocorreu incremento gradativo do número de esporos até 1993, que se estabilizou no terceiro cultivo, em 1994. A partir desse ano, não se detectaram diferenças significativas entre os períodos seco e chuvoso, em todos os tratamentos. Observou-se que a rotação da cultura da soja com a do milho não resultou em alterações significativas do número de esporos no solo, como também observado por Kurle \& Pfleger (1994) em áreas com plantio convencional de milho e soja em rotação.

A colonizaçao radicular das plantas foi elevada e não houve diferenças significativas entre as áreas com pastagem pura e consorciada contínuas, no período chuvoso do primeiro ano de cultivo em 1992 (Tabela 1). Entretanto, essa colonização radicular das forrageiras foi o dobro da observada nas plantas de soja. Dodd et al. (1990a) e Wilson \& Hartnett (1998) sugerem que essas diferenças podem estar relacionadas à fenologia das plantas, mas sobretudo à morfologia das raízes das forrageiras tropicais.

Nos cultivos seguintes, no período chuvoso, a colonização radicular das plantas forrageiras decresceu em 1993 e 1995 e cresceu, alternadamente, em 1994 e 1996 permanecendo, entretanto, abaixo do índice observado no primeiro ano. A redução da colonização radicular, após o primeiro ano, e sua estabilização com o tempo de cultivo indicam a importância da associação micorrízica na fase inicial do crescimento dessas plantas. Essa dependência micorrízica temporária de plantas forrageiras foi observada por outros autores (Howeler et al., 1987; Hartnett \& Wilson, 2002) que sugerem que a micorriza arbuscular é benéfica para essas plantas, somente sob condições de estresse, ou em fases específicas do ciclo de vida da planta. Efetivamente, Dodd et al. (1990a) constataram que não houve efeito da micorriza arbuscular na produção de braquiária (Brachiaria dictyoneura), com o tempo de cultivo, e comentam que respostas positivas dessa associação são esperadas na fase inicial de crescimento da planta, sobretudo para a sobrevivência das plântulas em um ecossistema agressivo como o das savanas.

No período seco, a colonização radicular das pastagens apresentou reduções, em relação ao período chuvoso, e permaneceu, em geral, estável durante os períodos do terceiro ao quinto cultivos. Staddon et al. (2003) sugerem que a resposta dos fungos micorrízicos arbusculares às mudanças climáticas, principalmente à seca, podem ser atribuídas diretamente a mudanças na densidade do micélio extra-radicular e, indiretamente, a mudanças na vegetação entre as estações, especialmente em relação à densidade e à abundância relativa de espécies de plantas. Na Região do Cerrado, durante a estação da seca, as atividades fisiológicas da maioria das plantas cultivadas reduzem-se drasticamente (Embrapa, 1998), exceto para o Stylosanthes guianensis cultivar Mineirão.

Nas áreas com lavoura contínua, a rotação das culturas de soja e milho beneficiou a colonização radicular da soja no quarto cultivo, em 1995, que foi significativamente mais elevada do que a observada nos dois primeiros anos de cultivo de soja. Esse dado mostra que o cultivo de plantas micorrízicas como o milho, no sistema de rotação, resulta em maior colonização radicular da cultura seguinte. Karasawa et al. (2002) constataram que após o cultivo de girassol (Helianthus annuus L.), planta micorrízica, o potencial de inóculo do solo aumentou e beneficiou a colonização radicular e o crescimento do milho subseqüente. Bever (2002) comenta, também, que a rotação de soja e milho é efetuada em certas partes dos Estados Unidos da América, para evitar decréscimos na produção após monoculturas repetitivas, e que o aumento na riqueza de espécies de fungos micorrízicos arbusculares é maior com cultivos repetitivos de milho, quando comparado ao observado com cultivos repetitivos de soja.

Nas áreas com rotação lavoura/pastagem e vice-versa, avaliadas somente no período chuvoso (Tabela 2), os tratamentos de lavoura e pastagem consorciada foram semelhantes aos apresentados na Tabela 1 até o quarto cultivo. A partir do terceiro cultivo, o número de esporos no solo se igualou, em geral, em todos os tratamentos, mantendo-se estável mesmo após a rotação ocorrida no quinto cultivo. A colonização radicular da pastagem consorciada e das culturas anuais foi semelhante à obser- 
vada nos sistemas contínuos, nos quatro anos de cultivo que antecederam a rotação. Contudo, no quinto cultivo após a rotação, a colonização radicular das plantas de milho introduzidas no sistema de pastagem foi mais elevada do que a observada nas plantas forrageiras, cultivadas antes da rotação, igualando-se ao porcentual verificado anteriormente, na área com lavoura contínua (Tabela 1), e ficando próximo do observado no terceiro cultivo do milho (1994), na área com rotação (Tabela 2). No sistema com lavoura inicial, a colonização radicular da forrageira após a rotação foi significativamente maior do que os índices observados no segundo e quarto cultivos do tratamento PC/LA e semelhante ao índice obtido no primeiro cultivo, nesse mesmo tratamento, e na pastagem consorciada contínua (Tabela 1). Esses dados enfatizam a importância da cultura utilizada no sistema de rotação, para beneficiar o estabelecimento dos fungos micorrízicos arbusculares, e a necessidade da micorriza arbuscular para as forrageiras, na fase inicial de seu cres- cimento e estabelecimento, conforme comentado também por outros autores (Hartnett et al., 1994; Hartnett \& Wilson, 2002).

A riqueza de espécies de fungos micorrízicos arbusculares no solo foi igualmente avaliada, tendo-se observado que a cultura utilizada e a prática de rotação influenciaram na composição dessa comunidade. A implantação da lavoura com culturas anuais favoreceu a multiplicação dos gêneros e de espécies de fungos micorrízicos arbusculares, em relação às pastagens pura ou consorciada (Tabela 3). Nessa última, a presença de leguminosas no consórcio beneficiou, também, o aparecimento de novos gêneros e de espécies de fungos em função do tempo de cultivo, conforme mostrado na avaliação de setembro de 1995, em relação ao tratamento com pastagem pura. Segundo Bever et al. (1996) e Eom et al. (2000), a composição da comunidade de plantas tem efeito significativo na diversidade e na composição da comunidade de fungos micorrízicos arbusculares.

Tabela 2. Esporos de fungos micorrízicos arbusculares nativos e colonização radicular das plantas hospedeiras, no período chuvoso, em solo com quatro anos de pastagem consorciada (PC), lavoura (LA) de soja (S) e milho (M) e após rotação recíproca no quinto ano $(1996)^{(1)}$.

\begin{tabular}{|c|c|c|c|c|}
\hline \multirow[t]{2}{*}{ Ano } & \multicolumn{2}{|c|}{ Esporos no solo (nº $/ 50 \mathrm{~g})$} & \multicolumn{2}{|c|}{ Colonização radicular (\%) } \\
\hline & $\mathrm{PC}$ & LA & $\mathrm{PC}$ & LA \\
\hline & 231 & $35 \mathrm{~S}$ & 65 & $26 \mathrm{~S}$ \\
\hline 1993 & 145 & $128 \mathrm{~S}$ & 31 & $49 \mathrm{~S}$ \\
\hline 1994 & 79 & $70 \mathrm{M}$ & 57 & $88 \mathrm{M}$ \\
\hline 1995 & 54 & $36 \mathrm{~S}$ & 41 & $60 \mathrm{~S}$ \\
\hline & LA & $\mathrm{PC}$ & LA & $\mathrm{PC}$ \\
\hline 1996 & $48 \mathrm{M}$ & 37 & $84 \mathrm{M}$ & 66 \\
\hline
\end{tabular}

(1)Os dados de esporos no solo foram transformados em $(x+0,5)^{0,5}$ e de colonização radicular em arc sen (x/100) ${ }^{0,5}$; a diferença mínima significativa a 5\% e o coeficiente de variação (\%) para número de esporos no solo foi 19 e 11,2, respectivamente; a diferença mínima significativa a 5\% e o coeficiente de variação (\%) para colonização radicular foi 12 e 10,7, respectivamente.

Tabela 3. Espécies de fungos micorrízicos arbusculares, em três épocas sucessivas de avaliação, no cerrado nativo (CN), nos experimentos iniciados em 1991 com pastagem pura (PP), pastagem consorciada (PC) e lavoura (LA) de soja e milho em rotação, e com rotação recíproca LA e PC no quinto ano $(1996)^{(1)}$.

\begin{tabular}{llll}
\hline Sistema & \multicolumn{3}{c}{ Época de amostragem } \\
\cline { 2 - 4 } & \multicolumn{1}{c}{$4 / 1994$} & \multicolumn{1}{c}{$9 / 1995$} & $4 / 1996$ \\
\hline CN & Asp. Lsp. Csp. & Asp. Lsp. Csp. & Asp. Lsp. Csp. \\
PP & Asp. Lsp. & Asp. Lsp. & Asp. Lsp. \\
PC & Asp. Lsp. & Asp. Lsp. Csp. & Asp. Lsp. Csp. Gsp. Esp. \\
LA & Asp. Lsp. Csp. Gsp. & Asp. Lsp. Csp. Gsp. & Asp. Lsp. Csp. Gsp. Esp. \\
PC/LA-Milho (1996) & Asp. Lsp. & Asp. Lsp. Csp. & Asp. Lsp. Csp. Gsp. Esp. \\
LA/PC (1996) & Asp. Lsp. Csp. Gsp. & Asp. Lsp. Csp. Gsp. & Asp. Lsp. Csp. Gsp. Esp. \\
\hline
\end{tabular}

(1)Espécies de fungos MA: Asp.: Acaulospora scrobiculata, A. mellea, A. tuberculata; Csp.: Scutellospora biornata, S. cerradensis, S. pellucida,

S. reticulata; Lsp.: Glomus occultum, G. clarum; Gsp.: Gigaspora gigantea, G. margarita; Esp.: Entrophospora colombiana. 
De acordo com Heijden et al. (2003), a diversidade de espécies fúngicas exerce um efeito importante na coexistência das plantas e na distribuição de $\mathrm{N}$ e P entre elas. Na área com rotação pastagem/lavoura, após a introdução do milho no quinto cultivo, o número de espécies de fungos micorrízicos arbusculares nativos ficou semelhante ao da área com cultura anual contínua. A presença simultânea de maior número de espécies de plantas na área de pastagem consorciada, ou em seqüência nas áreas com rotação teria, portanto, beneficiado essa comunidade, ao longo do tempo. Entretanto, essa variação pode resultar de uma seleção que ocorre em razão da especificidade observada entre plantas e fungos micorrízicos arbusculares (Bever et al., 1996; Heijden et al., 1998; Miranda \& Miranda, 2001).

O cerrado natural manteve o mesmo número de espécies ao longo do tempo, que foi inferior ao observado nas áreas cultivadas com culturas anuais e pastagem consorciada após cinco anos de cultivo. Dodd et al. (1990b) também observaram, em solos naturais das savanas colombianas, um menor número de espécies ao longo do tempo e em quantidade inferior às das áreas cultivadas. Além disso, Dodd et al. (1990a) também observaram que as espécies desses fungos, nativas de solos de savana, apresentam baixa a média eficiência para a absorção de nutrientes pelas plantas utilizadas, mostrando a importância do aumento da diversidade de espécies. Abbott \& Gazey (1994) sugerem que pode ser vantajoso manter altos níveis de diversidade de espécies de fungos micorrízicos arbusculares no solo, independentemente de sua contribuição individual para a formação da micorriza. Essa situação pode proporcionar uma oportunidade para espécies eficientes predominarem quando as condições do solo, modificadas com as práticas agrícolas, forem propícias. Segundo Heijden et al. (1998), o aumento na riqueza de espécies pode resultar no aumento da produtividade das culturas, uma vez que há maior possibilidade de se incluir um isolado muito eficiente para as plantas.

Abbott \& Gazey (1994) sugerem que as perturbações do solo causam modificações na predominância de espécies fúngicas, no solo, e que a diversidade de espécies seria menor em casos de perturbações mínimas, como observado nas pastagens contínuas neste trabalho. No caso de perturbações intermediárias, assim como observado nos tratamentos com cultura anual contínua em rotação, e com rotação pastagem/lavoura, essa diversidade é favorecida, como comentado por Oehl et al. (2003).

A identificação morfológica dos fungos micorrízicos arbusculares, nas amostras de campo, foram confirmadas pela observação dos esporos frescos desses fungos formados nas culturas armadilhas. Bever et al. (1996) obtiveram uma similaridade significativa na composição da comunidade desses fungos, entre análises feitas diretamente em amostras do campo, e amostras provenientes das culturas armadilhas. Foram verificadas 17 espécies, pertencentes a cinco gêneros (Tabela 3), das quais 16 puderam ser identificadas de acordo com as descrições prévias. Uma espécie, até então não descrita, foi posteriormente identificada como Scutellospora cerradensis (Spain \& Miranda, 1996) e depositada e registrada na INVAM (2004) e na BEG (2004).

Os gêneros Acaulospora e Glomus foram observados em todos os tratamentos (Tabela 3). O gênero Glomus tem sido encontrado, predominantemente em solos convencionalmente cultivados (Jansa et al., 2002; Oehl et al., 2003). Além desses gêneros, foi observada, na área com lavoura, a presença de espécies de Gigaspora (Gigaspora gigantea, Gigaspora margarita), que estavam ausentes nas áreas com pastagens contínuas, e do gênero Scutellospora. Esse último gênero, assim como os de Acaulospora e Glomus foram encontrados, também, nas áreas de cerrado nativo. Heijden et al. (2003) comentam que as variações nos efeitos sobre o crescimento das plantas pelas diferentes espécies de fungos micorrízicos arbusculares, parecem ser maiores quanto ao gênero do que quanto à espécie ou isolado, indicando que é mais provável observar efeitos complementares da diversidade de espécies quando diferentes gêneros desses fungos estão presentes no solo. A espécie Entrophospora colombiana ocorreu nas áreas cultivadas com cultura anual e pastagem consorciada e não foi encontrada no solo de cerrado nativo. Esses dados contrastam com os resultados obtidos por Jansa et al. (2002), que observaram a ausência de Scutellospora spp. e Entrophospora spp. em solos cultivados. Dodd et al. (1990b) observaram, contudo, número significativamente maior da espécie Entrophospora colombiana em solos cultivados com kudzu do que nos de savana.

Antes da rotação, foram encontradas espécies dos gêneros Acaulospora, Glomus e Scutellospora no sistema PC/LA e de Acaulospora, Glomus, Scutellospora e Gigaspora no sistema LA/PC (Tabela 3). Schenck \& Kinloch (1980) notaram que espécies de Gigaspora 
ocorreram com maior freqüência em áreas cultivadas com soja, enquanto nas pastagens houve maior número de Glomus e Acaulospora. Após a rotação, no quinto cultivo, a ocorrência desses quatro gêneros foi generalizada em ambos os sistemas de rotação. O gênero Entrophospora foi encontrado nesses tratamentos a partir do quinto cultivo, após a rotação das culturas, como observado nos cultivos contínuos de culturas anuais e pastagem consorciada.

Os dados da avaliação complementar, em casa de vegetação, da contribuição dos fungos micorrízicos no crescimento de plantas de soja e de capimandropógon, utilizados nos sistemas de cultivo em campo, são apresentados na Tabela 4. A produção de matéria seca dessas culturas aumentou e foi significativamente maior em presença da micorriza, evidenciando a dependência micorrízica dessas plantas para o seu crescimento. Os fungos micorrízicos arbusculares nativos promoveram um acréscimo médio de $53 \%$ e $95 \%$ de matéria seca, respectivamente, de soja e Andropogon gayanus, conforme verificado por Howeler et al. (1987). Esses autores, ao comparar a dependência micorrízica de várias culturas anuais e forrageiras, observaram que Andropogon gayanus foi uma das gramíneas forrageiras mais dependentes da micorriza arbuscular, apesar de sua densidade radicular ser maior. Sieverding (1991) realça a importância da utilização de plantas dependentes da micorriza arbuscular, na

Tabela 4. Matéria seca da parte aérea (MS), esporos no solo (E), colonização radicular (CR) e dependência micorrízica (DM) de soja e Andropogon gayanus, em casa de vegetação, cultivados no solo coletado no quinto ano de lavoura (LA) e de pastagem pura (PP), com e sem fungos micorrízicos arbusculares nativos ${ }^{(1)}$.

\begin{tabular}{lcccc}
\hline Micorriza & MS $\left(\mathrm{g} \mathrm{vaso}^{-1}\right)$ & $\mathrm{E}\left(\mathrm{n}^{\circ} / 50 \mathrm{~g}\right)$ & $\mathrm{CR}(\%)$ & $\mathrm{DM}(\%)^{(2)}$ \\
\hline \multicolumn{5}{c}{ Soja (LA) } \\
Com & $5,28 \mathrm{a}$ & \multicolumn{5}{c}{$56 \mathrm{a}$} & $86 \mathrm{a}$ & 53 \\
Sem & $2,50 \mathrm{~b}$ & $0 \mathrm{~b}$ & $0 \mathrm{~b}$ & - \\
\hline $\mathrm{CV}(\%)$ & 6,2 & 8,3 & 5,9 \\
\hline \multicolumn{5}{c}{ Capim-andropógon (PP) } \\
Com & $0,84 \mathrm{a}$ & $24 \mathrm{a}$ & $57 \mathrm{a}$ & 95 \\
Sem & $0,04 \mathrm{~b}$ & $0 \mathrm{~b}$ & $0 \mathrm{~b}$ & - \\
\hline $\mathrm{CV}(\%)$ & 7,3 & 8,6 & 5,9 \\
\hline
\end{tabular}

(1) Os dados de esporos no solo foram transformados em $(x+0,5)^{0,5}$ e de colonização radicular, fósforo e nitrogênio no tecido, em arc sen $(\mathrm{x} / 100)^{0,5}$; médias seguidas da mesma letra, na coluna e dentro de cada cultura, não diferem significativamente a $5 \%$ de probabilidade, pelo teste de Duncan. ${ }^{(2)} \mathrm{DM}=[$ (MS com micorriza - MS sem micorriza)/MS com micorriza] x 100 . rotação de culturas dos sistemas de produção, e menciona que a seqüência adequada de plantas pode aumentar o potencial de inóculo do solo ou favorecer a multiplicação de espécies de fungos micorrízicos arbusculares que podem ser eficientes para as culturas posteriores. Deve-se ressaltar, entretanto, que, nesse experimento, fez-se a comparação entre solo natural (com micorriza), e o mesmo solo esterilizado a vapor e com filtrados do solo natural, sem os fungos micorrízicos, semelhante ao efetuado em outros trabalhos (Bever, 2002; Heijden et al., 2003; Oehl et al., 2003). Segundo Koide \& Li (1989), essa metodologia poderia não ser totalmente apropriada, uma vez que os filtrados e o solo em si podem não conter as mesmas proporções das populações de microrganismos. Hartnett et al. (1994) comentam, entretanto, que plantas cultivadas em solo sem micorriza mostraram crescimento idêntico às cultivadas em solo pasteurizado e adicionado de uma suspensão de solo não esterilizado, para retornar outros componentes da microbiota.

Os dados apresentados enfatizam a necessidade de se considerar a micorriza arbuscular ao se manejar os agrossistemas e programar práticas agrícolas, como a rotação de culturas, para manter a sua abundância e diversidade.

\section{Conclusões}

1. Altas taxas de colonização micorrízica nas raízes das plantas em solo natural, em relação à ausência delas em solo esterilizado a vapor, indicam que os fungos micorrízicos arbusculares são determinantes para o crescimento das culturas nos sistemas de plantio com culturas anuais e pastagens, e o número de esporos desses fungos nativos varia em função da cultura utilizada, estações seca e chuvosa e tempo de cultivo.

2. As rotações das culturas, soja e milho e soja, milho e pastagens, no sistema de produção aumentam o número de esporos e a diversidade de espécies dos fungos micorrízicos arbusculares nativos no solo.

3. As pastagens puras e consorciadas beneficiam a multiplicação de esporos dos fungos micorrízicos arbusculares no solo, principalmente na fase inicial de implantação e no consórcio com leguminosas, e a presença de diversas plantas hospedeiras no sistema aumenta a diversidade de espécies de fungos micorrízicos arbusculares no solo, ao longo do tempo de cultivo. 


\section{Referências}

ABBOTT, L.K.; GAZEY, C. An ecological view of the formation of VA mycorrhizas. Plant and Soil, v.159, p.69-78, 1994.

ABBOTT, L.K.; ROBSON, A.D. The impact of agricultural practices on mycorrhizal fungi. In: PANKHURST, C.E.; DOUBE, B.M.; GUPTA, V.V.S.R.; GRACE, P.R. (Ed.). Soil biota: management in sustainable farming systems. Victoria, Australia: CSIRO, 1994. p.88-95.

BEG. Banque Européenne des Glomales. Disponível em: <http:/ /www.kent.ac.uk/bio/beg>. Acesso em: dez. 2004.

BEVER, J.D. Host-specificity of AM fungal populations growth rates can generate feedback on plant growth. Plant and Soil, v.244, p.281-290, 2002.

BEVER, J.D.; MORTON, J.B.; ANTONOVICS, J.; SCHULTZ, P.A. Host-dependent sporulation and species diversity of arbuscular mycorrhizal fungi in a mown grassland. Journal of Ecology, v.84, p.71-82, 1996.

COOLEN, W.R. Methods for the extraction of Meloidogyne spp. and other nematodes from roots and soil. In: LAMBERTI, F.; TAYLOR, C.E. (Ed.). Root-knot nematodes (Meloidogyne species): systematics, ecology and control. London: Academic, 1979. p.317-329.

DODD, J.C.; ARIAS, I.; KOOMEN, I.; HAYMAN, D.S. The management of populations of vesicular-arbuscular mycorrhizal fungi in acid-infertile soils of a savanna ecosystem. I. The effect of precropping and inoculation with VAM-fungi on plant growth and nutrition in the field. Plant and Soil, v.122, p.229-240, 1990a.

DODD, J.C.; ARIAS, I.; KOOMEN, I.; HAYMAN, D.S. The management of populations of vesicular-arbuscular mycorrhizal fungi in acid-infertile soils of a savanna ecosystem. II. The effects of precrops on the spore populations of native and introduced VAMfungi. Plant and Soil, v.122, p.241-247, 1990b.

EMBRAPA. Serviço Nacional de Levantamento e Conservação de Solos (Rio de Janeiro, RJ). Manual de métodos de análises de solo. Rio de Janeiro, 1979. Não paginado.

EMBRAPA. Centro de Pesquisa Agropecuária dos Cerrados (Planaltina, DF). Estabelecimento e utilização do estilosantes mineirão. Planaltina, DF: Embrapa-CPAC, 1998. 6p. (Comunicado técnico, 74).

EOM, A.H.; HARTNETT, D.C.; WILSON, G.W.T. Host plant species effects on arbuscular mycorrhizal fungal communities in tallgrass prairie. Oecologia, v.122, p.435-444, 2000.

GERDEMANN, J.W.; NICOLSON, T.H. Spores of mycorrhizal endogone species extracted from soil by wet sieving and decanting. Transactions of the British Mycological Society, v.46, p.235244, 1963.

GIOVANNETTI, M.; MOSSE, B. An evaluation of techniques to measuring vesicular arbuscular mycorrhizal infection in roots. New Phytologist, v.84, p.489-500, 1980.

HARTNETT, D.C.; WILSON, G.W.T. The role of mycorrhizas in plant community structure and dynamics: lessons from grasslands. Plant and Soil, v.244, p.319-331, 2002.
HARTNETT, D.C.; SAMENUS, R.J.; FISCHER, L.E.; HETRICK, B.A.D. Plant demographic responses to mycorrhizal symbiosis in tallgrass prairie. Oecologia, v.99, p.21-26, 1994.

HEIJDEN, M.G.A. van der; KLIRONOMOS, J.N.; URSIC, M.; MOUTOGLIS, P.; STREITWOLF-ENGEL, R.; BOLLER, T.; WIEMKEN, A.; SANDERS, I.R. Mycorrhizal fungal diversity determines plant biodiversity, ecosystem variability and productivity. Nature, v.396, p.69-72, 1998.

HEIJDEN, M.G.A. van der; WIEMKEN, A.; SANDERS, I.R. Different arbuscular mycorrhizal fungi alter coexistence and resource distribution between co-ocurring plant. New Phytologist, v.157, p.569-578, 2003.

HOWELER, R.H.; SIEVERDING, E.; SAIF, S. Practical aspects of mycorrhizal technology in some tropical crops and pastures. Plant and Soil, v.100, p.249-283, 1987.

INVAM. International Culture Collection of Arbuscular and Vesicular-Arbuscular Mycorrhizal Fungi. Disponível em: <http:/ /www.invam.caf.wvu.edu>. Acesso em: dez. 2004.

JANSA, J.; MOZAFAR, A.; ANKEN, T.; RUH, R.; SANDERS, I.R.; FROSSARD, E. Diversity and structure of AMF communities as affected by tillage in a temperate soil. Mycorrhiza, v.12, p.225234, 2002.

KARASAWA, T.; KASAHARA, Y.; TAKEBE, M. Differences in growth responses of maize to preceding cropping caused by fluctuation in the population of indigenous arbuscular mycorrhizal fungi. Soil Biology and Biochemistry, v.34, p.851857, 2002.

KOIDE, R.T.; LI, M. Appropriate controls for vesiculararbuscular mycorrhiza research. New Phytologist, v.111, p.35-44, 1989.

KURLE, J.E.; PFLEGER, F.L. Arbuscular mycorrhizal fungus spore populations respond to conversions between low-input and conventional management practices in a corn-soybean rotation. Agronomy Journal, v.86, p.467-475, 1994.

MIRANDA, J.C.C.; MIRANDA, L.N. Seleção e recomendação de uso de espécies de fungos micorrízicos arbusculares. Planaltina: Embrapa Cerrados, 2001. 3p. (Comunicado técnico, 52).

MORTON, J.B.; BENTIVENGA, S.P.; WHEELER, W.W. Germplasm in the International Collection of Arbuscular and Vesiculararbuscular Mycorrhizal Fungi (INVAM) and procedures for culture development, documentation, and storage. Mycotaxon, v.48, p.491528, 1993.

OEHL, F.; SIEVERDING, E.; INEICHEN, K.; MADER, P.; BOLLER, T.; WIEMKEN, A. Impact of land use intensity on the species diversity of arbuscular mycorrhizal fungi in agroecosystems of Central Europe. Applied and Environmental Microbiology, v.69, p.2816-2824, 2003.

PHILLIPS, J.M.; HAYMAN, D.S. Improved procedures for clearing roots and stainning parasitic and vesicular-arbuscular mycorrhyzal fungi for rapid assessment of infection. Transactions of the British Mycological Society, v.55, p.158-161, 1970. 
SAS INSTITUTE (Cary, Estados Unidos). Statistical analysis systems user's guide: version 6. $4^{\text {th }}$ ed. Cary, NC: SAS, 1989.

SCHENCK, N.C.; KINLOCH, R.A. Incidence of mycorrhizal fungi on six field crops in monoculture on a newly cleared woodland site. Mycologia, v.72, p.445-456, 1980.

SCHENCK, N.C.; PÉREZ, Y. Manual for the identification of VA mycorrhizal fungi. $3^{\text {rd }}$ ed. Gainesville: Synergistic, 1990. 286p.

SIEVERDING, E. Vesicular-arbuscular mycorrhiza management in tropical agrosystems. Eschborn: Deutsche Gesellschaft für Technische Zusammenarbeit (GTZ), Federal Republic of Germany, 1991. 371p.

SIQUEIRA, J.O.; MOREIRA, F.M.S. Microbial populations and activities in highly-weathered acidic soils: highlights of the Brazilian research. In: MONIZ, A.C.; FURLANI, A.M.C.; SCHAFFERT,
R.E.; FAGERIA, N.K.; ROSOLEM, C.A.; CANTARELLA, H. (Ed.). Plant-soil interactions at low $\mathbf{p H}$ : sustainable agriculture and forestry production. Campinas: Brazilian Soil Science Society, 1997. p.139-156.

SPAIN, J.L.; MIRANDA, J.C.C. Scutellospora cerradensis: an ornamented species in the Gigasporaceae (Glomales) from the Cerrado region of Brazil. Mycotaxon, v.60, p.129-136, 1996.

STADDON, P.L.; THOMPSON, K.; JAKOBSEN, I.; GRIME, J.P.; ASKEW, A.P.; FITTER, A.H. Mycorrhizal fungal abundance as affected by long-term climatic manipulations in the field. Global Change Biology, v.9, p.186-194, 2003.

WILSON, G.W.T.; HARTNETT, D.C. Interspecific variation in plant responses to mycorrhizal colonization in prairie grasses and forbs. American Journal of Botany, v.85, p.1732-1738, 1998.

Recebido em 29 de março de 2004 e aprovado em 23 de março de 2005 